Processes' and 'Severe Storms and Hail' are devoted to more macroscopic aspects of cloud physics. Various precipitation systems are described, attention being given to the airflow which governs the release and distribution of rainfall. A brief discussion is presented of the efficiency with which cloud water is transformed into precipitation. A detailed account follows of the dynamics and life cycle of severe storms. This leads naturally to a description of the formation of hail and the energy balance equations which govern the growth of hail pellets.

The final two chapters contain skeletal descriptions of current works in the important areas of weather modification and numerical modelling of cloud processes.

The problems for students presented at the end of each chapter are imaginatively devised and well balanced. It is helpful that in this second edition answers are given to odd-numbered questions. The book is modestly priced and serves as an excellent introduction to cloud physics.

J. Latham is Head of the Physics Department of UMIST, Manchester, UK, and President of the International Commission on Atmospheric Electricity.

\section{Inorganic analysis}

\section{F.J.C. Rossotti}

Vogel's Textbook of Macro and Semimicro Qualitative Inorganic Analysis. By G. Svehla. (Longman: Harlow, UK, 1979.) £14.95. Practical Inorganic Chemistry. By G. Pass and H. Sutcliffe. Pp.242. (Chapman and Hall: London, 1979.) Paperback £3.95.

Four editions of Vogel's textbook appeared between 1937 and 1954, the heyday of qualitative inorganic analysis in British schools and universities. Twentyfive years later, Svehla has produced a fifth edition, in an age when less time is devoted to practical chemistry in British curricula and the popularity of systematic qualitative analysis has waned. One of the benefits of a sound grounding in qualitative analysis is that students become familiar with a great deal of phenomenological inorganic chemistry, for example, colours, solubilities and reactions in solution. A backwards swing of the pendulum would now be timely.

The systematic qualitative analysis of some 44 cations and 47 anions is described. Length and contents of the new edition are much the same as before. Two short chapters on interfering anions and on paper chromatography in the previous edition have been assimilated into other chapters and there is some shuffling of order. However, updating seems to have been modest. Thus the section on solvent extraction remains weak and largely irrelevant in its detailed content. It might, for example, have been helpful to students to inform them that systems of qualitative analysis, which rely entirely on solvent extraction, now exist. In this connection, citation of key references (as in the Vogel Quantitative Inorganic text) would have been helpful in much of the book.

The most disappointing feature of the new edition is the still antiquated treatment of reactions in solution. Thus ammonia and copper (II) ions do not yield a unique tetra-ammine (Dawson, 1900) but rather five mononuclear ammines in stepwise equilibrium (Bjerrum, 1931). The bloodred colour produced on mixing iron (III) and thiocyanate ions has been variously attributed to $\mathrm{Fe}(\mathrm{NCS})_{6}{ }^{3-}$ (third edition), $\mathrm{Fe}(\mathrm{NCS})^{2+}$ (fourth edition) and now to $\mathrm{Fe}(\mathrm{NCS})_{3}$ (fifth edition). In fact, six thiocyanato complexes coexist in stepwise equilibrium and in relative amounts which depend upon the free thiocyanate ion concentration. Discharge of the red colour by fluoride ions is not due to the formation of a unique complex $\mathrm{FeF}_{6}{ }^{3-}$, but rather to an array of mononuclear fluoro complexes. Moreover, it is doubtful whether the hexafluoro species exists at all in aqueous solution. These typical outdated misconceptions about the nature of interactions between inorganic species in aqueous solution seriously mar an otherwise useful bench-book.

By contrast, Pass and Sutcliffe's text deals with the practical inorganic chemistry that has so largely replaced qualitative analysis in British university curricula. The current volume is a paperback reprint of the second edition of 1974. It contains a wide variety of preparative exercises, together with some reactions of transition metal ions and applications of some instrumental techniques. The interweaving of a certain amount of theory with the experimental instructions, together with the modest length and price of the book, must make it attractive for many courses.

Arbitrary choices of topic are inevitable if space is at a premium, but the ornission of solvent extraction from a chapter on separation techniques, which contains ionexchange, seems somewhat surprising. Again, it is regrettable that instruction on Job's method of continuous variations persists in the 2 nd edition. This archaic method is of very limited applicability, again by virtue of stepwise complex formation.

F.J.C. Rossotti is a Lecturer in Inorganic Chemistry at the University of Oxford, UK.

\section{Plasma physics}

\section{I.H. Hutchinson}

Physics of High Temperature Plasmas. Second edition. By G. Schmidt. Pp.408. (Academic: New York, 1979.) \$29.50.

THE dilemma of most didactic texts, particularly in relatively rapidly developing subjects, lies between a presentation of the historical development of the discipline and an essentially axiomatic exposition along what are seen as the most logical lines. Few general plasma physics texts follow as completely the latter, nonhistorical approach as the second edition of Physics of High Temperature Plasmas by George Schmidt. This bias is emphasised by the deletion of the first edition's quite detailed bibliographies accompanying each chapter, in favour of a very brief list of books at the end. However, perhaps because of this approach, the new edition gives very little evidence of ageing.

The coverage is very wide, including treatments of particle motion guidingcentre approximations, the derivation of the CGL approximation, two chapters on magnetohydrodynamics, Vlasov wave and instability analyses, bounded plasma effects and touching on relaxation and collisions. The bulk of the material new to this edition is contained in a chapter on non-linear waves, providing a good introduction to topics not often covered by general texts.

The book is fully committed to being self-contained in its development. In this it succeeds rather well, hardly every specifically appealing to the reader's credence ("it can be shown"). However, there are definitely occasions when rigour is sacrificed in claiming the self-evidence of statements which, on reflection, are plausible though not obvious. Such points are inevitable and not necessarily detrimental. Indeed, greater attention to minutiae might have made the exposition too formal. As it is, while the many examples that are worked out in depth are mostly of direct experimental relevance, the uninitiated are left wondering just how the results really work out with a laboratory plasma. The feeling is enhanced by the fact that what few illustrations there are of the results in action are mostly of computational simulations.

A remarkable success of this book is that it covers such a broad range of topics with significant depth in just four hundred pages. This is achieved, on the one hand, by a very happy choice of examples and lines of development of the subject. On the other hand, though, the prose is compact, sometimes terse, and the mathematical 